Supplementary information

\title{
The basal ganglia control the detailed kinematics of learned motor skills
}

In the format provided by the authors and unedited 
Supplementary information

The basal ganglia control the detailed kinematics of learned motor skills 


\section{Supplementary table 1: Lesion coordinates DLS, DMS, GPi}

Injection coordinates were (in mm, according to Paxinos Brain Atlas):

\begin{tabular}{|l|l|l|l|l|l|l|l|l|l|}
\hline & \multicolumn{2}{|l}{ DLS } & \multicolumn{2}{l|}{ DMS } & \multicolumn{2}{l|}{ GPi } \\
\hline AP* & +0.7 & +0.7 & -0.3 & -0.3 & +1.2 & +1.2 & +0.2 & +0.2 & -2.3 \\
\hline ML* & \pm 3.6 & \pm 3.6 & \pm 4.0 & \pm 4.0 & \pm 1.9 & \pm 1.9 & \pm 1.9 & \pm 1.9 & \pm 2.8 \\
\hline DV & -5.5 & -4.5 & -5 & -4 & -5.5 & -4.5 & -5.5 & -4.5 & -7.5 \\
\hline
\end{tabular}

* measured from bregma 
Supplementary table 2: Lesion coordinates motor cortex

Injection coordinates were (in mm, according to Paxinos Brain Atlas):

\begin{tabular}{|c|c|c|c|c|c|c|}
\hline & \multicolumn{6}{|c|}{$M C$} \\
\hline$P^{*}$ & +1 & +1 & +1 & +1 & +3 & +3 \\
\hline$M L^{*}$ & \pm 2 & \pm 2 & \pm 4.0 & \pm 4.0 & \pm 2 & \pm 2 \\
\hline DV & -1.6 & -0.8 & -1.6 & -0.8 & -1.6 & -0.8 \\
\hline
\end{tabular}

* measured from bregma 


\section{Supplementary table 3: Projection tracing coordinates}

Injection coordinates were (in mm, according to Paxinos Brain Atlas):

\begin{tabular}{|c|c|c|c|c|c|c|c|}
\hline & \multicolumn{5}{|l|}{$M C$} & \multicolumn{2}{|l|}{ PFC } \\
\hline$A P^{*}$ & +1 & +1 & -1.5 & -2.25 & +3 & +3.2 & +2.2 \\
\hline$M L^{*}$ & \pm 2 & \pm 4 & \pm 2.75 & \pm 2.5 & \pm 2 & \pm 0.6 & \pm 0.6 \\
\hline DV & -1.5 to -0.7 & -1.5 to -0.7 & -1.5 to -0.7 & -1.5 to -0.7 & -1.5 to -0.7 & -4.4 to -2.0 & -4.4 to -2.0 \\
\hline
\end{tabular}

* measured from bregma 


\section{Supplementary table 4: Statistical Results for Figure 6}

\begin{tabular}{|c|c|c|c|c|}
\hline Figure & Description & Samples & Test & Results \\
\hline \multirow{2}{*}{ Fig. 6D } & \multirow{2}{*}{$\begin{array}{l}\text { JS divergence between } \\
\text { IPI and ITI for DLS- } \\
\text { lesioned animals across } \\
\text { time points (early, pre- } \\
\text { lesion, post-lesion) }\end{array}$} & \multirow{2}{*}{7 rats } & $\begin{array}{l}\text { Repeated measures } \\
\text { ANOVA. Factor 1: } \\
\text { Time point }\end{array}$ & $\begin{array}{l}\text { Time point: } \\
F(2,12)=200.37 \\
P<0.0001\end{array}$ \\
\hline & & & $\begin{array}{l}\text { Post-hoc test: } \\
\text { Bonferroni }\end{array}$ & $\begin{array}{l}\text { P values: } \\
\text { 1)Post vs. Pre: } P<0.001 \\
\text { 2) Early vs. Pre: } P<0.001 \\
\text { 3)Early vs. Post: } P=0.921\end{array}$ \\
\hline \multirow[b]{2}{*}{ Fig. 6D } & \multirow{2}{*}{$\begin{array}{l}\text { JS divergence between } \\
\text { IPI and ITI for DMS- } \\
\text { lesioned animals across } \\
\text { time points (early, pre- } \\
\text { lesion, post-lesion) }\end{array}$} & \multirow[b]{2}{*}{5 rats } & $\begin{array}{l}\text { Repeated measures } \\
\text { ANOVA. Factor 1: } \\
\text { Time point }\end{array}$ & $\begin{array}{l}\text { Time point: } \\
F(2,8)=14.88, \\
P=0.0182\end{array}$ \\
\hline & & & $\begin{array}{l}\text { Post-hoc test: } \\
\text { Bonferroni }\end{array}$ & $\begin{array}{l}\text { P values: } \\
\text { 1)Post vs. Pre: } P>0.99 \\
\text { 2)Early vs. Pre: } P=0.005 \\
\text { 3) Early vs. Post: } P=0.004\end{array}$ \\
\hline \multirow[b]{2}{*}{ Fig. 6D } & \multirow{2}{*}{$\begin{array}{l}\text { JS divergence between } \\
\text { IPI and ITI for Control } \\
\text { animals across time } \\
\text { points (early, pre-lesion, } \\
\text { post-lesion) }\end{array}$} & \multirow[b]{2}{*}{5 rats } & $\begin{array}{l}\text { Repeated measures } \\
\text { ANOVA. Factor 1: } \\
\text { Time point }\end{array}$ & $\begin{array}{l}\text { Time point: } \\
F(2,8)=15.53 \\
P=0.0169\end{array}$ \\
\hline & & & $\begin{array}{l}\text { Post-hoc test: } \\
\text { Bonferroni }\end{array}$ & $\begin{array}{l}\text { P values: } \\
\text { 1)Post vs. Pre: } P>0.99 \\
\text { 2) Early vs. Pre: } P=0.003 \\
\text { 3) Early vs. Post: } P=0.007\end{array}$ \\
\hline
\end{tabular}




\section{Supplementary table 5: Statistical Results for Figure 7}

\begin{tabular}{|c|c|c|c|c|}
\hline Figure & Description & Samples & Test & Results \\
\hline \multirow{2}{*}{ Fig. 7A } & \multirow{2}{*}{$\begin{array}{l}\text { Comparison of trial-to-trial } \\
\text { pairwise correlations within } \\
\text { animals after DLS lesion across } \\
\text { time points (pre-lesion to pre- } \\
\text { lesion, post-lesion to post- } \\
\text { lesion, pre-lesion to post-lesion) }\end{array}$} & \multirow{2}{*}{6 rats } & $\begin{array}{l}\text { Repeated } \\
\text { measures ANOVA. } \\
\text { Factor 1: Time } \\
\text { point }\end{array}$ & $\begin{array}{l}\text { Time point: } F(2,10)=9.84 \text {, } \\
P=0.0258\end{array}$ \\
\hline & & & $\begin{array}{l}\text { Post-hoc test: } \\
\text { Bonferroni }\end{array}$ & $\begin{array}{l}\text { P values: } \\
\text { 1)Pre vs. Post: } P>0.99 \\
\text { 2)Pre vs. Pre-Post: } P=0.008 \\
\text { 3)Post vs. Pre-Post: } P=0.013\end{array}$ \\
\hline Fig. 7B & $\begin{array}{l}\text { Comparison of trial-to-trial } \\
\text { pairwise correlations within } \\
\text { animals after DMS lesion across } \\
\text { time points (pre-lesion to pre- } \\
\text { lesion, post-lesion to post- } \\
\text { lesion, pre-lesion to post-lesion) }\end{array}$ & 5 rats & $\begin{array}{l}\text { Repeated } \\
\text { measures ANOVA. } \\
\text { Factor 1: Time } \\
\text { point }\end{array}$ & $\begin{array}{l}\text { Time point: } \\
F(2,7)=0.26 \\
P=0.6377\end{array}$ \\
\hline \multirow{2}{*}{ Fig. 7C } & \multirow{2}{*}{$\begin{array}{l}\text { Comparison of trial-to-trial } \\
\text { pairwise correlations across } \\
\text { animals after DLS lesion across } \\
\text { time points (pre-lesion to pre- } \\
\text { lesion, post-lesion to post- } \\
\text { lesion, pre-lesion to post-lesion) }\end{array}$} & \multirow{2}{*}{6 rats } & $\begin{array}{l}\text { Repeated } \\
\text { measures ANOVA. } \\
\text { Factor 1: Time } \\
\text { point }\end{array}$ & $\begin{array}{l}\text { Time point: } F(2,10)=46.99 \\
P=0.001\end{array}$ \\
\hline & & & $\begin{array}{l}\text { Post-hoc test: } \\
\text { Bonferroni }\end{array}$ & $\begin{array}{l}\text { P values: } \\
\text { 1)Pre vs. Post: } P<0.001 \\
\text { 2)Pre vs. Pre-Post: } P>0.99 \\
\text { 3)Post vs. Pre-Post: } P<0.001\end{array}$ \\
\hline Fig. 7D & $\begin{array}{l}\text { Comparison of trial-to-trial } \\
\text { pairwise correlations across } \\
\text { animals after DMS lesion across } \\
\text { time points (pre-lesion to pre- } \\
\text { lesion, post-lesion to post- } \\
\text { lesion, pre-lesion to post-lesion) }\end{array}$ & 5 rats & $\begin{array}{l}\text { Repeated } \\
\text { measures ANOVA. } \\
\text { Factor 1: Time } \\
\text { point }\end{array}$ & $\begin{array}{l}\text { Time point: } F(2,7)=0.75 \text {, } \\
P=0.4358\end{array}$ \\
\hline
\end{tabular}




\section{Supplementary table 6: Statistical Results for Figure 8}

\begin{tabular}{|c|c|c|c|c|}
\hline Figure & Description & Samples & Test & Results \\
\hline \multirow[b]{2}{*}{ Fig. 8A } & \multirow{2}{*}{$\begin{array}{l}\text { Comparisons of trial-to- } \\
\text { trial pairwise correlations } \\
\text { between lever-press } \\
\text { trajectories within } \\
\text { animals with DLS lesions } \\
\text { across conditions: } \\
\text { correlations between the } \\
\text { same presses (1st to 1st, } \\
\text { 2nd to 2nd - intra-press) } \\
\text { and between different } \\
\text { presses (1st to } 2 \text { nd - } \\
\text { inter-press) were each } \\
\text { compared pre- and post- } \\
\text { lesion. Further, all presses } \\
\text { were compared between } \\
\text { the pre and post } \\
\text { conditions (all 1st and all } \\
\text { 2nd - pre-post). }\end{array}$} & \multirow[b]{2}{*}{6 rats } & $\begin{array}{l}\text { Repeated } \\
\text { measures } \\
\text { ANOVA. } \\
\text { Factor 1: } \\
\text { Condition }\end{array}$ & $\begin{array}{l}\text { Condition: } \\
F(4,20)=0.0002 \\
P=0.0287\end{array}$ \\
\hline & & & $\begin{array}{l}\text { Post-hoc } \\
\text { test: } \\
\text { Bonferroni }\end{array}$ & $\begin{array}{l}P \text { values: } \\
\text { 1) Intra pre vs. Intra post: } P>0.99 \\
\text { 2) Intra pre vs. Inter pre: } P=0.001 \\
\text { 3) Intra pre vs. Inter post: } P>0.99 \\
\text { 4) Intra pre vs. All: } P=0.021 \\
\text { 5) Intra post vs. Inter pre: } P=0.002 \\
\text { 6) Intra post vs. Inter post: } P>0.99 \\
\text { 7) Intra post vs. All: } P=0.038 \\
\text { 8) Inter pre vs. Inter post: } P=0.005 \\
\text { 9) Inter pre vs. All: } P>0.99 \\
\text { 10) Inter post vs. All: } P=0.083\end{array}$ \\
\hline \multirow{2}{*}{ Fig. 8B } & \multirow{2}{*}{$\begin{array}{l}\text { Comparisons of trial-to- } \\
\text { trial pairwise correlations } \\
\text { between lever-press } \\
\text { trajectories for animals } \\
\text { with DLS lesions across } \\
\text { time points (pre to pre, } \\
\text { post to post, pre to post) }\end{array}$} & \multirow{2}{*}{$\begin{array}{l}\text { for each } \\
\text { comparison: } \\
6 \text { rats with } \\
2 \text { lever- } \\
\text { presses: } \\
6 * 2 * 6 * 2= \\
144\end{array}$} & $\begin{array}{l}\text { Repeated } \\
\text { measures } \\
\text { ANOVA. } \\
\text { Factor 1: } \\
\text { Time point }\end{array}$ & $\begin{array}{l}\text { Time point: } \\
F(2,22)=23.31 \\
P=0.0005\end{array}$ \\
\hline & & & $\begin{array}{l}\text { Post-hoc } \\
\text { test: } \\
\text { Bonferroni }\end{array}$ & $\begin{array}{l}\text { P values: } \\
\text { 1)Pre vs. Post: } P<0.001 \\
\text { 2)Pre vs. Pre-Post: } P=0.409 \\
\text { 3)Post vs. Pre-Post: } P<0.001\end{array}$ \\
\hline Fig. 8C & $\begin{array}{l}\text { Comparisons of trial-to- } \\
\text { trial pairwise correlations } \\
\text { between lever-press } \\
\text { trajectories for animals } \\
\text { with DLS lesions across } \\
\text { time points (early to } \\
\text { early, post to post, early } \\
\text { to post) }\end{array}$ & $\begin{array}{l}\text { for each } \\
\text { comparison: } \\
4 \text { rats with } \\
2 \text { lever- } \\
\text { presses: } \\
4 * 2 * 4 * 2= \\
64\end{array}$ & $\begin{array}{l}\text { Repeated } \\
\text { measures } \\
\text { ANOVA. } \\
\text { Factor 1: } \\
\text { Time point }\end{array}$ & $\begin{array}{l}\text { Time point: } \\
F(2,14)=3.86 \\
P=0.0902\end{array}$ \\
\hline
\end{tabular}




\section{Supplementary table 7: Statistical Results for Extended Data Figure 6}

\begin{tabular}{|l|l|l|l|l|}
\hline Figure & Description & Samples & Test & Results \\
\hline Fig. ED 6A & $\begin{array}{l}\text { Comparison of mean } \\
\text { IPI before and after } \\
\text { DLS lesion }\end{array}$ & 3 rats & $\begin{array}{l}\text { Two-tailed } \\
\text { paired t-test }\end{array}$ & $\begin{array}{l}\text { P value: } \\
P=0.393\end{array}$ \\
\hline Fig. ED 6A & $\begin{array}{l}\text { Comparison of mean } \\
\text { CV before and after } \\
\text { DLS lesion }\end{array}$ & 3 rats & $\begin{array}{l}\text { Two-tailed } \\
\text { paired t-test }\end{array}$ & $\begin{array}{l}\text { P value: } \\
P=0.906\end{array}$ \\
\hline Fig. ED 6A & $\begin{array}{l}\text { Comparison of IPI } \\
\text { close to target before } \\
\text { and after DLS lesion }\end{array}$ & 3 rats & $\begin{array}{l}\text { Two-tailed } \\
\text { paired t-test }\end{array}$ & $\begin{array}{l}\mathrm{P} \text { value: } \\
\mathrm{P}=0.106\end{array}$ \\
\hline Fig. ED 6A & $\begin{array}{l}\text { Comparison of mean } \\
\text { ITI before and after } \\
\text { DLS lesion }\end{array}$ & 3 rats & $\begin{array}{l}\text { Two-tailed } \\
\text { paired t-test }\end{array}$ & $\begin{array}{l}\mathrm{P} \text { value: } \\
\mathrm{P}=0.359\end{array}$ \\
\hline
\end{tabular}




\section{Supplementary table 8: Statistical Results for Extended Data Figure 7}

\begin{tabular}{|c|c|c|c|c|}
\hline Figure & Description & Samples & Test & Results \\
\hline Fig. ED 7 & \multicolumn{4}{|c|}{$\begin{array}{l}\text { Comparisons of performance measures in DLS-lesioned, DMS-lesioned or Control animals } \\
\text { across time points: early in training, pre-lesion, post-lesion and late after lesion }\end{array}$} \\
\hline \multirow[b]{2}{*}{ Fig. ED 7} & \multirow[b]{2}{*}{ IPI; DLS } & \multirow[b]{2}{*}{7 rats } & $\begin{array}{l}\text { Repeated measures ANOVA. } \\
\text { Factor 1: Time Point }\end{array}$ & $\begin{array}{l}\text { Time Point: } \\
F(3,18)=9.57, P=0.0213\end{array}$ \\
\hline & & & Post-hoc test: Bonferroni & $\begin{array}{l}P \text { values: } \\
\text { 1)Early vs. Pre: } P<0.001 \\
\text { 2)Early vs. Post: } P>0.99 \\
\text { 3)Early vs. Late: } P=0.686 \\
\text { 4)Pre vs. Post: } P=0.005 \\
\text { 5)Pre vs. Late: } P=0.018 \\
\text { 6)Post vs. Late: } P>0.99\end{array}$ \\
\hline \multirow[b]{2}{*}{ Fig. ED 7} & \multirow[b]{2}{*}{ CV; DLS } & \multirow[b]{2}{*}{7 rats } & $\begin{array}{l}\text { Repeated measures ANOVA. } \\
\text { Factor 1: Time Point }\end{array}$ & $\begin{array}{l}\text { Time Point: } \\
F(3,18)=12.38, P=0.0125\end{array}$ \\
\hline & & & Post-hoc test: Bonferroni & $\begin{array}{l}P \text { values: } \\
\text { 1)Early vs. Pre: } P<0.001 \\
\text { 2)Early vs. Post: } P>0.99 \\
\text { 3)Early vs. Late: } P=0.41 \\
\text { 4)Pre vs. Post: } P=0.001 \\
\text { 5)Pre vs. Late: } P=0.011 \\
\text { 6)Post vs. Late: } P>0.99\end{array}$ \\
\hline \multirow[b]{2}{*}{ Fig. ED 7} & \multirow[b]{2}{*}{$\begin{array}{l}\text { IPI close to } \\
\text { target; DLS }\end{array}$} & \multirow[b]{2}{*}{7 rats } & $\begin{array}{l}\text { Repeated measures ANOVA. } \\
\text { Factor 1: Time Point }\end{array}$ & $\begin{array}{l}\text { Time Point: } \\
F(3,18)=62.74, P=0.0002\end{array}$ \\
\hline & & & Post-hoc test: Bonferroni & $\begin{array}{l}P \text { values: } \\
\text { 1)Early vs. Pre: } P<0.001 \\
\text { 2)Early vs. Post: } P>0.99 \\
\text { 3)Early vs. Late: } P>0.99 \\
\text { 4)Pre vs. Post: } P<0.001 \\
\text { 5)Pre vs. Late: } P<0.001 \\
\text { 6)Post vs. Late: } P=0.927\end{array}$ \\
\hline \multirow[b]{2}{*}{ Fig. ED 7} & \multirow[b]{2}{*}{ ITI; DLS } & \multirow[b]{2}{*}{7 rats } & $\begin{array}{l}\text { Repeated measures ANOVA. } \\
\text { Factor 1: Time Point }\end{array}$ & $\begin{array}{l}\text { Time Point: } \\
F(3,18)=4.87, P=0.0406\end{array}$ \\
\hline & & & Post-hoc test: Bonferroni & $\begin{array}{l}P \text { values: } \\
\text { 1)Early vs. Pre: } P=0.008 \\
\text { 2)Early vs. Post: } P=0.679 \\
\text { 3)Early vs. Late: } P=0.639 \\
\text { 4)Pre vs. Post: } P=0.275 \\
\text { 5)Pre vs. Late: } P=0.294 \\
\text { 6)Post vs. Late: } P>0.99\end{array}$ \\
\hline
\end{tabular}




\begin{tabular}{|c|c|c|c|c|}
\hline \multirow[b]{2}{*}{ Fig. ED 7} & \multirow[b]{2}{*}{$\begin{array}{l}\text { Lever } \\
\text { presses/session; } \\
\text { DLS }\end{array}$} & \multirow[b]{2}{*}{7 rats } & $\begin{array}{l}\text { Repeated measures ANOVA. } \\
\text { Factor 1: Time Point }\end{array}$ & $\begin{array}{l}\text { Time Point: } \\
F(3,18)=4.88, P=0.0424\end{array}$ \\
\hline & & & Post-hoc test: Bonferroni & $\begin{array}{l}P \text { values: } \\
\text { 1)Early vs. Pre: } P=0.016 \\
\text { 2)Early vs. Post: } P>0.99 \\
\text { 3)Early vs. Late: } P>0.99 \\
\text { 4)Pre vs. Post: } P=0.048 \\
\text { 5)Pre vs. Late: } P=0.091 \\
\text { 6)Post vs. Late: } P>0.99\end{array}$ \\
\hline \multirow[b]{2}{*}{ Fig. ED 7} & \multirow[b]{2}{*}{ IPI; DMS } & \multirow[b]{2}{*}{5 rats } & $\begin{array}{l}\text { Repeated measures ANOVA. } \\
\text { Factor 1: Time Point }\end{array}$ & $\begin{array}{l}\text { Time Point: } \\
F(3,12)=12.32, P=0.0247\end{array}$ \\
\hline & & & Post-hoc test: Bonferroni & $\begin{array}{l}P \text { values: } \\
\text { 1)Early vs. Pre: } P=0.003 \\
\text { 2)Early vs. Post: } P=0.001 \\
\text { 3)Early vs. Late: } P=0.003 \\
\text { 4)Pre vs. Post: } P>0.99 \\
\text { 5)Pre vs. Late: } P>0.99 \\
\text { 6)Post vs. Late: } P>0.99\end{array}$ \\
\hline \multirow[b]{2}{*}{ Fig. ED 7} & \multirow[b]{2}{*}{ CV; DMS } & \multirow[b]{2}{*}{5 rats } & $\begin{array}{l}\text { Repeated measures ANOVA. } \\
\text { Factor 1: Time Point }\end{array}$ & $\begin{array}{l}\text { Time Point: } \\
F(3,12)=7.84, P=0.0488\end{array}$ \\
\hline & & & Post-hoc test: Bonferroni & $\begin{array}{l}P \text { values: } \\
\text { 1)Early vs. Pre: } P=0.011 \\
\text { 2)Early vs. Post: } P=0.025 \\
\text { 3)Early vs. Late: } P=0.007 \\
\text { 4)Pre vs. Post: } P>0.99 \\
\text { 5)Pre vs. Late: } P>0.992 \\
\text { 6) Post vs. Late: } P>0.99\end{array}$ \\
\hline \multirow[b]{2}{*}{ Fig. ED 7} & \multirow[b]{2}{*}{$\begin{array}{l}\text { IPI close to } \\
\text { target; DMS }\end{array}$} & \multirow[b]{2}{*}{5 rats } & $\begin{array}{l}\text { Repeated measures ANOVA. } \\
\text { Factor 1: Time Point }\end{array}$ & $\begin{array}{l}\text { Time Point: } \\
F(3,12)=15.74, P=0.0166\end{array}$ \\
\hline & & & Post-hoc test: Bonferroni & $\begin{array}{l}P \text { values: } \\
\text { 1)Early vs. Pre: } P=0.001 \\
\text { 2)Early vs. Post: } P=0.002 \\
\text { 3)Early vs. Late: } P<0.001 \\
\text { 4)Pre vs. Post: } P>0.99 \\
\text { 5)Pre vs. Late: } P>0.99 \\
\text { 6)Post vs. Late: } P>0.99\end{array}$ \\
\hline \multirow[b]{2}{*}{ Fig. ED 7} & \multirow[b]{2}{*}{ ITI; DMS } & \multirow[b]{2}{*}{5 rats } & $\begin{array}{l}\text { Repeated measures ANOVA. } \\
\text { Factor 1: Time Point }\end{array}$ & $\begin{array}{l}\text { Time Point: } \\
F(3,18)=13.42, P=0.0215\end{array}$ \\
\hline & & & Post-hoc test: Bonferroni & $\begin{array}{l}P \text { values: } \\
\text { 1) Early vs. Pre: } P=0.001 \\
\text { 2)Early vs. Post: } P=0.001 \\
\text { 3)Early vs. Late: } P=0.0013 \\
\text { 4)Pre vs. Post: } P>0.99 \\
\text { 5)Pre vs. Late: } P>0.99 \\
\text { 6)Post vs. Late: } P>0.99\end{array}$ \\
\hline
\end{tabular}




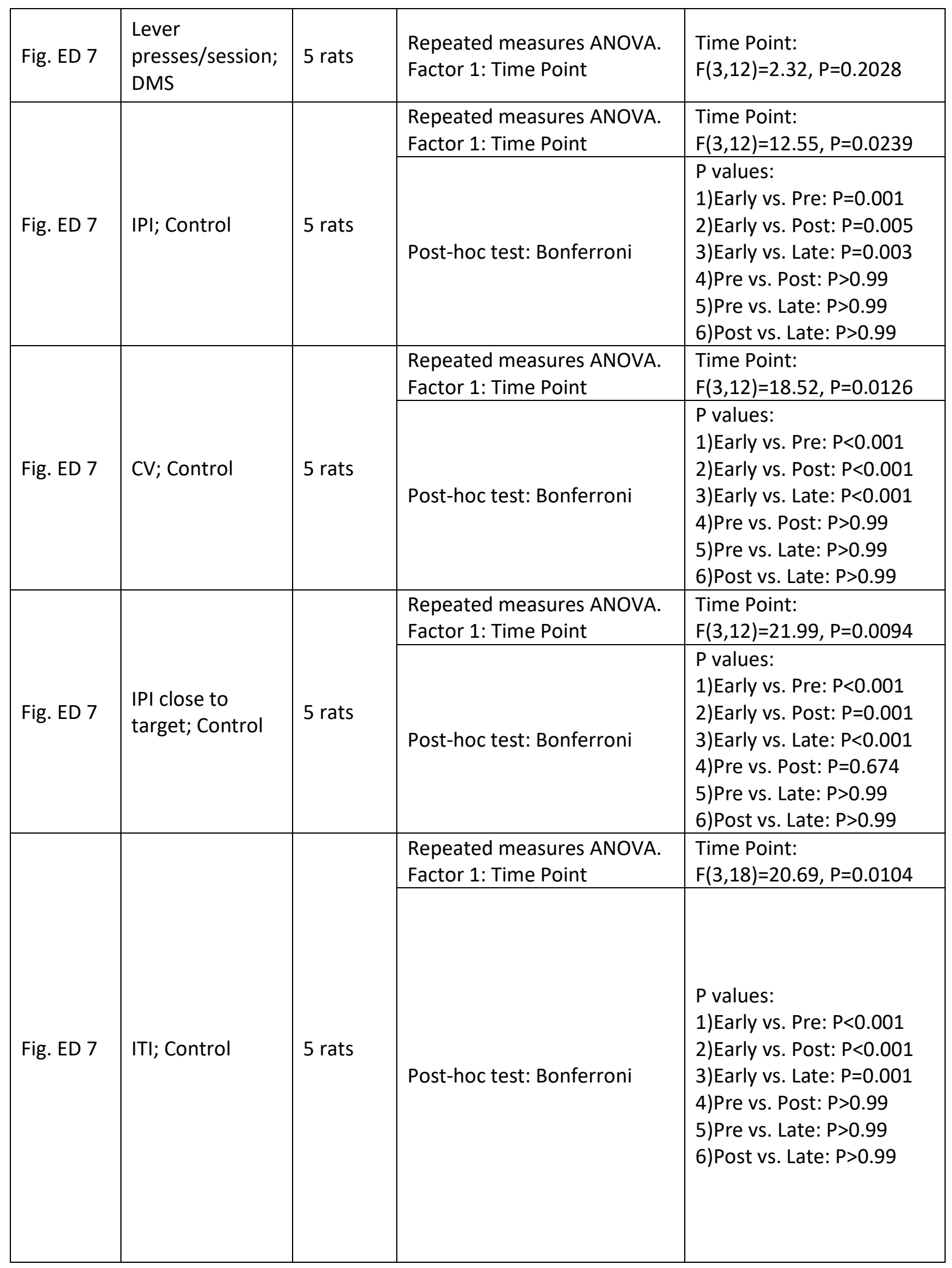




\begin{tabular}{|c|c|c|c|c|}
\hline & & & $\begin{array}{l}\text { Repeated measures ANOVA. } \\
\text { Factor 1: Time Point }\end{array}$ & $\begin{array}{l}\text { Time Point: } \\
F(3,12)=5.20, P=0.0480\end{array}$ \\
\hline Fig. ED 7 & $\begin{array}{l}\text { Lever } \\
\text { presses/session; } \\
\text { Control }\end{array}$ & 5 rats & Post-hoc test: Bonferroni & $\begin{array}{l}P \text { values: } \\
\text { 1)Early vs. Pre: } P=0.131 \\
\text { 2) Early vs. Post: } P=0.043 \\
\text { 3)Early vs. Late: } P=0.024 \\
\text { 4)Pre vs. Post: } P>0.99 \\
\text { 5)Pre vs. Late: } P>0.99 \\
\text { 6) Post vs. Late: } P>0.99\end{array}$ \\
\hline
\end{tabular}




\section{Supplementary table 9: Statistical Results for Extended Data Figure 8}

\begin{tabular}{|c|c|c|c|c|}
\hline Figure & Description & Samples & Test & Results \\
\hline Fig. ED 8B & \multicolumn{4}{|c|}{$\begin{array}{l}\text { Comparisons of performance measures in GPi-lesioned animals across time points: } \\
\text { early in training, pre-lesion, post-lesion and late after lesion; DLS data and statistics } \\
\text { reproduced from Extended Data Figure } 7\end{array}$} \\
\hline \multirow[b]{2}{*}{ Fig. ED 8B } & \multirow[b]{2}{*}{ IPI; GPi } & \multirow[b]{2}{*}{5 rats } & $\begin{array}{l}\text { Repeated measures ANOVA. } \\
\text { Factor 1: Time Point }\end{array}$ & $\begin{array}{l}\text { Time: } \\
F(3,12)=8.8 \\
P=0.0413\end{array}$ \\
\hline & & & Post-hoc test: Bonferroni & $\begin{array}{l}\text { P values: } \\
\text { 1)Early vs. Pre: } P=0.004 \\
\text { 2) Early vs. Post: } P=0.029 \\
\text { 3) Early vs. Late: } P=0.006 \\
\text { 4)Pre vs. Post: } P>0.99 \\
\text { 5) Pre vs. Late: } P>0.99 \\
\text { 6) Post vs. Late: } P>0.99\end{array}$ \\
\hline \multirow[b]{2}{*}{ Fig. ED 8B } & \multirow[b]{2}{*}{ CV; Gpi } & \multirow[b]{2}{*}{5 rats } & $\begin{array}{l}\text { Repeated measures ANOVA. } \\
\text { Factor 1: Time Point }\end{array}$ & $\begin{array}{l}\text { Time: } \\
F(3,12)=4.77 \\
P=0.0206\end{array}$ \\
\hline & & & Post-hoc test: Bonferroni & $\begin{array}{l}\text { P values: } \\
\text { 1)Early vs. Pre: } P=0.018 \\
\text { 2)Early vs. Post: } P>0.99 \\
\text { 3)Early vs. Late: } P=0.34 \\
\text { 4)Pre vs. Post: } P=0.228 \\
\text { 5)Pre vs. Late: } P=0.817 \\
\text { 6) Post vs. Late: } P>0.99\end{array}$ \\
\hline \multirow[b]{2}{*}{ Fig. ED 8B } & \multirow[b]{2}{*}{$\begin{array}{l}\text { IPI close to } \\
\text { target; GPi }\end{array}$} & \multirow[b]{2}{*}{5 rats } & $\begin{array}{l}\text { Repeated measures ANOVA. } \\
\text { Factor 1: Time Point }\end{array}$ & $\begin{array}{l}\text { Time: } \\
F(3,12)=12.92 \\
P=0.0229\end{array}$ \\
\hline & & & Post-hoc test: Bonferroni & $\begin{array}{l}\text { P values: } \\
\text { 1)Early vs. Pre: } P<0.001 \\
\text { 2)Early vs. Post: } P=0.948 \\
\text { 3)Early vs. Late: } P=0.39 \\
\text { 4)Pre vs. Post: } P=0.005 \\
\text { 5)Pre vs. Late: } P=0.012 \\
\text { 6) Post vs. Late: } P>0.99\end{array}$ \\
\hline \multirow[b]{2}{*}{ Fig. ED 8B } & \multirow[b]{2}{*}{ ITI; GPi } & \multirow[b]{2}{*}{5 rats } & $\begin{array}{l}\text { Repeated measures ANOVA. } \\
\text { Factor 1: Time Point }\end{array}$ & $\begin{array}{l}\text { Time: } \\
F(3,12)=6.72 \\
P=0.0195\end{array}$ \\
\hline & & & Post-hoc test: Bonferroni & $\begin{array}{l}\text { P values: } \\
\text { 1)Early vs. Pre: } P=0.006 \\
\text { 2)Early vs. Post: } P>0.99 \\
\text { 3)Early vs. Late: } P=0.878 \\
\text { 4)Pre vs. Post: } P=0.038 \\
\text { 5) Pre vs. Late: } P=0.111 \\
\text { 6) Post vs. Late: } P>0.99\end{array}$ \\
\hline
\end{tabular}




\begin{tabular}{|l|l|l|l|l|}
\hline \multirow{2}{*}{ Fig. 8D } & $\begin{array}{l}\text { JS divergence } \\
\text { between IPI } \\
\text { and ITI for } \\
\text { GPi-lesioned } \\
\text { animals across } \\
\text { time points } \\
\text { Fig. 8D }\end{array}$ & 5 rats & $\begin{array}{l}\text { Repeated measures ANOVA. } \\
\text { Factor 1: Time point }\end{array}$ & $\begin{array}{l}\text { Time point: } \\
\mathrm{F}(2,10)=9.89, \\
\mathrm{P}=0.0255\end{array}$ \\
\cline { 2 - 4 } & $\begin{array}{l}\text { (early, pre- } \\
\text { lesion, post- } \\
\text { lesion) }\end{array}$ & Post-hoc test: Bonferroni & $\begin{array}{l}\text { P values: } \\
\text { 1)Post vs. Pre: } \mathrm{P}=0.034 \\
\text { 2)Early vs. Pre: } \mathrm{P}=0.005 \\
\text { 3)Early vs. Post: } \mathrm{P}=0.754\end{array}$ \\
\hline
\end{tabular}




\section{Supplementary table 10: Statistical Results for Extended Data Figure 9}

\begin{tabular}{|c|c|c|c|c|}
\hline Figure & Description & Samples & Test & Results \\
\hline Fig. ED 9A & $\begin{array}{l}\text { Comparison of mean } \\
\text { lever-press speed } \\
\text { before and after DLS } \\
\text { lesion }\end{array}$ & 6 rats & $\begin{array}{l}\text { Two-tailed } \\
\text { paired t-test }\end{array}$ & $\begin{array}{l}P \text { value: } \\
P=0.813\end{array}$ \\
\hline Fig. ED 9A & $\begin{array}{l}\text { Comparison of peak } \\
\text { lever-press speed } \\
\text { before and after DLS } \\
\text { lesion }\end{array}$ & 6 rats & $\begin{array}{l}\text { Two-tailed } \\
\text { paired t-test }\end{array}$ & $\begin{array}{l}P \text { value: } \\
P=0.779\end{array}$ \\
\hline \multirow{2}{*}{ Fig. ED 9B } & \multirow{2}{*}{$\begin{array}{l}\text { Comparisons of trial- } \\
\text { to-trial pairwise } \\
\text { correlations } \\
\text { between lever-press } \\
\text { trajectories for } \\
\text { animals with DLS } \\
\text { lesions across time } \\
\text { points (early to } \\
\text { early, post to post, } \\
\text { early to post) }\end{array}$} & \multirow{2}{*}{$\begin{array}{l}\text { for each } \\
\text { comparison: } 6 \\
\text { rats with } 2 \\
\text { lever-presses: } \\
6 * 2 * 6 * 2= \\
144\end{array}$} & $\begin{array}{l}\text { Repeated } \\
\text { measures } \\
\text { ANOVA. } \\
\text { Factor 1: } \\
\text { Time point }\end{array}$ & $\begin{array}{l}\text { Time point: } \\
F(2,22)=11.97 \\
P=0.005\end{array}$ \\
\hline & & & $\begin{array}{l}\text { Post-hoc } \\
\text { test: } \\
\text { Bonferroni }\end{array}$ & $\begin{array}{l}\text { P values: } \\
\text { 1)Early vs. Post: } P<0.001 \\
\text { 2)Early vs. Early-Post: } P=0.091 \\
\text { 3)Post vs. Early-Post: } P=0.051\end{array}$ \\
\hline
\end{tabular}




\section{Supplementary table 11: Statistical Results for Extended Data Figure 10}

\begin{tabular}{|c|c|c|c|c|}
\hline Figure & Description & Samples & Test & Results \\
\hline Fig. ED10F & $\begin{array}{l}\text { Comparison of trial-to- } \\
\text { trial pairwise correlations } \\
\text { of trajectories across } \\
\text { animals with small DLS } \\
\text { lesions between time } \\
\text { points (pre-lesion to pre- } \\
\text { lesion, post-lesion to } \\
\text { post-lesion, pre-lesion to } \\
\text { post-lesion) }\end{array}$ & 3 rats & $\begin{array}{l}\text { Repeated } \\
\text { measures } \\
\text { ANOVA. } \\
\text { Factor 1: } \\
\text { Time point }\end{array}$ & $\begin{array}{l}\text { Time point: } \\
F(2,4)=3.18, \\
P=0.1493\end{array}$ \\
\hline \multirow[b]{2}{*}{ Fig. ED10I } & \multirow{2}{*}{$\begin{array}{l}\text { Comparisons of trial-to- } \\
\text { trial pairwise correlations } \\
\text { between lever-press } \\
\text { trajectories for animals } \\
\text { with large DLS lesions } \\
\text { early and post-lesion and } \\
\text { animals with small DLS } \\
\text { lesions post-lesion. }\end{array}$} & \multirow{2}{*}{$\begin{array}{l}\text { For early and } \\
\text { for post: } 6 \\
\text { rats with } 2 \\
\text { lever-presses } \\
\text { each = } \\
12 * 12=144 \\
\text { comparisons; } \\
\text { for small vs. } \\
\text { early or vs. } \\
\text { post: } 3 \text { rats * } \\
2 \text { presses * } 6 \\
\text { rats } 2 \\
\text { presses }=72 \\
\text { comparisons }\end{array}$} & $\begin{array}{l}\text { Repeated } \\
\text { measures } \\
\text { ANOVA. } \\
\text { Factor 1: } \\
\text { Condition }\end{array}$ & $\begin{array}{l}\text { Condition: } \\
F(4,44)=76.63, \\
P<0.0001\end{array}$ \\
\hline & & & $\begin{array}{l}\text { Post-hoc } \\
\text { test: } \\
\text { Bonferroni }\end{array}$ & $\begin{array}{l}\text { P values: } \\
\text { 1)Early vs. Post: } P<0.001 \\
\text { 2) Early vs. small-early: } P<0.001 \\
\text { 3)Early vs. small-post: } P<0.001 \\
\text { 4)Post vs.small-early: } P<0.001 \\
\text { 5)Post vs.small-post: } P<0.001 \\
\text { 6) small-early vs. small-post: } \\
P>0.991\end{array}$ \\
\hline
\end{tabular}

